\title{
Visualization of Long Noncoding RNA MEG3 in Living Cells by Triple-Helix-Powered 3D catcher
}

\author{
Hua Yang, ${ }^{\dagger}, \#$ Yufei Zhang, ${ }^{\dagger, \#}$ Xuan Xu, ${ }^{\star}$ Huanxiang Wang, ${ }^{\dagger}$ Ziyun Huang, ${ }^{\dagger}$ Ziling \\ Luo, ${ }^{\dagger}$ Xiangxi Deng, ${ }^{\dagger}$ Qian Xue,${ }^{\dagger}$ Zhihe Qing, ${ }^{\dagger}$ Zhen Zou, ${ }^{\dagger}, *$ Ronghua Yang ${ }^{\dagger, *}$ \\ ${ }^{\dagger}$ Hunan Provincial Key Laboratory of Cytochemistry, School of Chemistry and Food \\ Engineering, Changsha University of Science and Technology, Changsha 410114, P. \\ R. China;
}

Children's Medical Center, People's Hospital of Hunan Province, Changsha, Hunan 410002, P. R. China.

*To whom correspondence should be addressed:

E-mail: $\underline{\text { Yangrh@pku.edu.cn; }}$ Fax: +86-731-88822523. 
Table S1. Sequences of oligonucleotides used in this work.

\begin{tabular}{|c|c|c|}
\hline Name & $\begin{array}{l}\text { Oligo } \\
\text { DNA }\end{array}$ & Sequences(5'-3') \\
\hline \multirow{4}{*}{ 3D catcher } & $\begin{array}{c}\mathrm{a} \\
(83-\mathrm{nt})\end{array}$ & $\begin{array}{l}\text { BHQ2/TCCTCTGCTGGGCGCCCCAGCTCTCCTATA } \\
\text { TTATTGAGCCGCGCCCAAGGCCAGCGTTCCAAG } \\
\text { GCGAATACTAGATCATTACT/Cy5 }\end{array}$ \\
\hline & $\begin{array}{c}\mathrm{b} \\
(83-\mathrm{nt})\end{array}$ & $\begin{array}{l}\text { Cy5/CAAGGACATCAGTGGACGGATGATGTCATC } \\
\text { GAACCCTGACTACCAGAGACCCGGGTAAGAGG } \\
\text { GAAAATATAGGAGATAGAGGA/BHQ2 }\end{array}$ \\
\hline & $\begin{array}{c}\mathrm{c} \\
(55-\mathrm{nt})\end{array}$ & $\begin{array}{l}\text { CGCTCCCTCTCTGCTCTAACGATGACATTGTCCT } \\
\text { TGCGAGTAAGATAAGCGTATT }\end{array}$ \\
\hline & $\underset{(55-n t)}{d}$ & $\begin{array}{l}\text { AGAGCAGAGAGGGAGCGAACCTTGGAATCGGC } \\
\text { TCAATATCCCTCTTAAGTCAGGG }\end{array}$ \\
\hline \multirow[t]{2}{*}{ Sequence of DTWH } & a1 & $\begin{array}{l}\text { BHQ2/TCCTCTATCTCCTATATTATTGAGCCGCGC } \\
\text { CCAAGGCCAGCGTTCCAAGGCGAATACTAGATC } \\
\text { ATTACT/Cy5 }\end{array}$ \\
\hline & $\mathrm{b} 1$ & $\begin{array}{l}\text { Cy5/CAAGGACAATGTCATCGAACCCTGACTTAA } \\
\text { GAGGGAAAATATAGGAGATAGAGGA/BHQ2 }\end{array}$ \\
\hline \multirow[t]{2}{*}{ Sequence of CDT } & $\mathrm{c} 1$ & $\begin{array}{l}\text { ACACACGAGTGTGGTCAAACGATGACATTGTCC } \\
\text { TTGCGAGTAATGATCTAGTATT }\end{array}$ \\
\hline & $\mathrm{d} 1$ & $\begin{array}{l}\text { TGACCACACTCGTGTGTAACCTTGGAATCGGCT } \\
\text { CAATATCCCTCTTAAGTCAGGG }\end{array}$ \\
\hline $\begin{array}{l}\text { DNA mimics } \\
\quad(129-\mathrm{nt})\end{array}$ & & $\begin{array}{l}\text { AGAGCAGAGAGGGAGCGCGCCTTGGCTCGCTG } \\
\text { GCCTTGGCGGCGGCTCCTCAGGAGAGCTGGGG } \\
\text { CGCCCACGAGAGGATCCCTCACCCGGGTCTCTC } \\
\text { CTCAGGGATGACATCATCCGTCCACCTCCTTG }\end{array}$ \\
\hline M1 & & $\begin{array}{l}\text { AGAGCAGAGAGGGAGCGCGCCTTGGCTCGCTG } \\
\text { GCCTTGGCGGCGGCTCCTCAGGAGATAATCCAC } \\
\text { ATTAGCGAGAGGATCCCTCACCCGGGTCTCTCC } \\
\text { TCAGGGATGACATCATCCGTCCACCTCCTTG }\end{array}$ \\
\hline M2 & & $\begin{array}{l}\text { AGAGCAGAGAGGGAGCGCGCCTTGGCTCGCTG } \\
\text { GCCTTGGCGGCGGCTCCTCAGGAGATAATCCAC } \\
\text { ATTAGCGAGAGGATCCCTCTATACCTACTAACCT } \\
\text { CAGGGATGACATCATCCGTCCACCTCCTTG }\end{array}$ \\
\hline M3 & & $\begin{array}{l}\text { AGAGCAGAGAGGGAGCGCGCCTTGGCTCGCTG } \\
\text { GCCTTGGCGGCGGCTCCTCAGGAGATAATCCAC } \\
\text { ATTAGCGAGAGGATCCCTCTATACCTACTAACCT } \\
\text { CAGGGATGACAAGTTACAGGATACTCCTTG }\end{array}$ \\
\hline MnSOD mRNA & & AAUCAACUGGGAGAAUGUAACUG \\
\hline miRNA-21 & & UAGCUUAUCAGACUGAUGUUGA \\
\hline miRNA-205 & & ACCAGAUUUCAGUGGAGUGAAGUUCAGG \\
\hline double-stranded DNA & & AGTGCTTCAGCTACGACTCGCATGAAGCACT \\
\hline
\end{tabular}




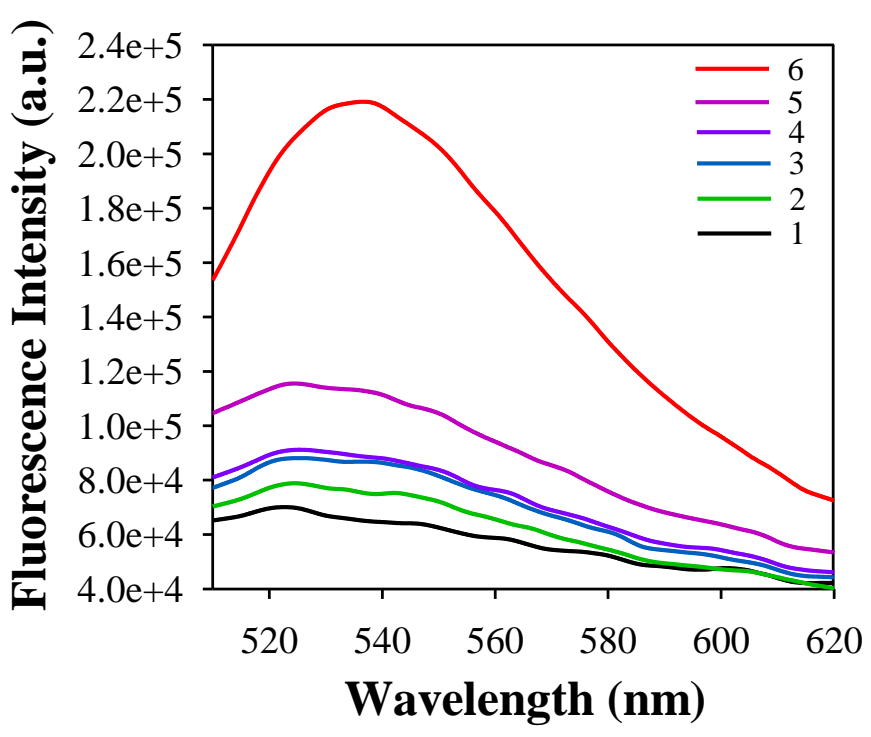

Figure S1. Fluorescence intensity of different oligonucleotides added to $2 \mathrm{mM}$ fisetin (FIS). 1. blank; 2. DNA mimics; 3. control DNA tetrahedrons (CDT) probe; 4. 3D catcher; 5. CDT + DNA mimics; 6. 3D catcher + DNA mimics. The fluorescence intensity was measured at $375 \mathrm{~nm}$.

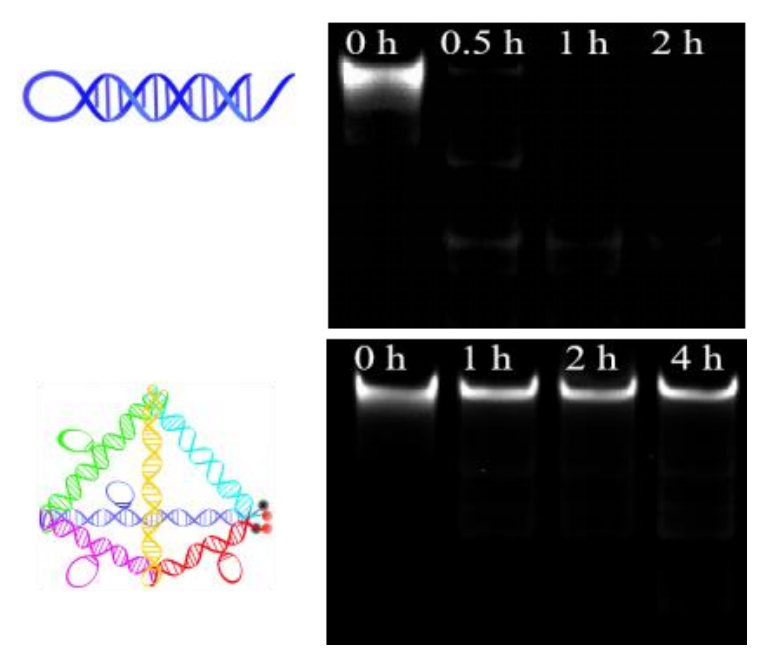

Figure S2. Electrophoresis characterization for the degradation of 3D catcher and double-stranded DNA treated with Dnase I ( $3 \mathrm{U} / \mathrm{mL})$ at $37{ }^{\circ} \mathrm{C}$ under various times by using PAGE (10\%). 


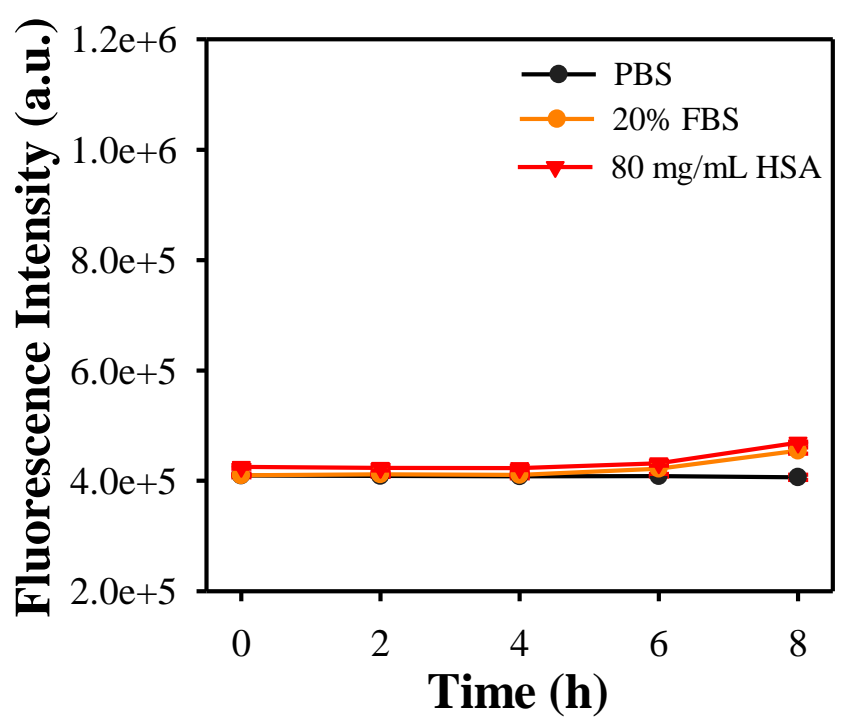

Figure S3. Stability analysis of 3D catcher in PBS, 20\% FBS and $80 \mathrm{mg} / \mathrm{mL}$ HSA. The solution was incubated at $37^{\circ} \mathrm{C}$ for different times, and then excited at $640 \mathrm{~nm}$ and received fluorescence intensity at $668 \mathrm{~nm}$.

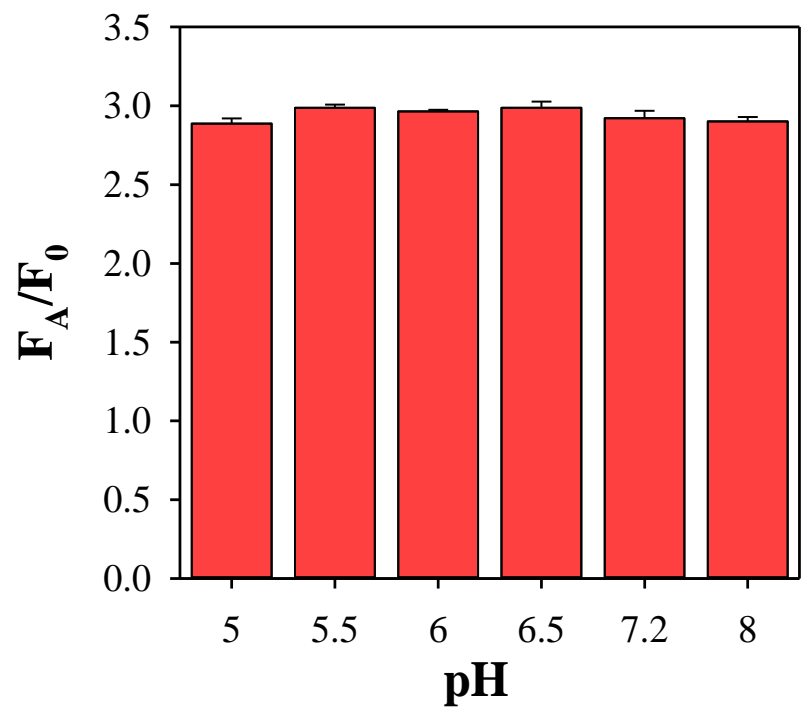

Figure S4. The effect of different $\mathrm{pH}$ values $(5.0,5.5,6,6.6,7.2,8.0)$ on the reaction system in PBS buffer solution. The signal gain is indicated by $F_{A} / F_{0}, F_{A}$ is the emission fluorescence intensity at $668 \mathrm{~nm}$ and $\mathrm{F}_{0}$ is the background fluorescence. 


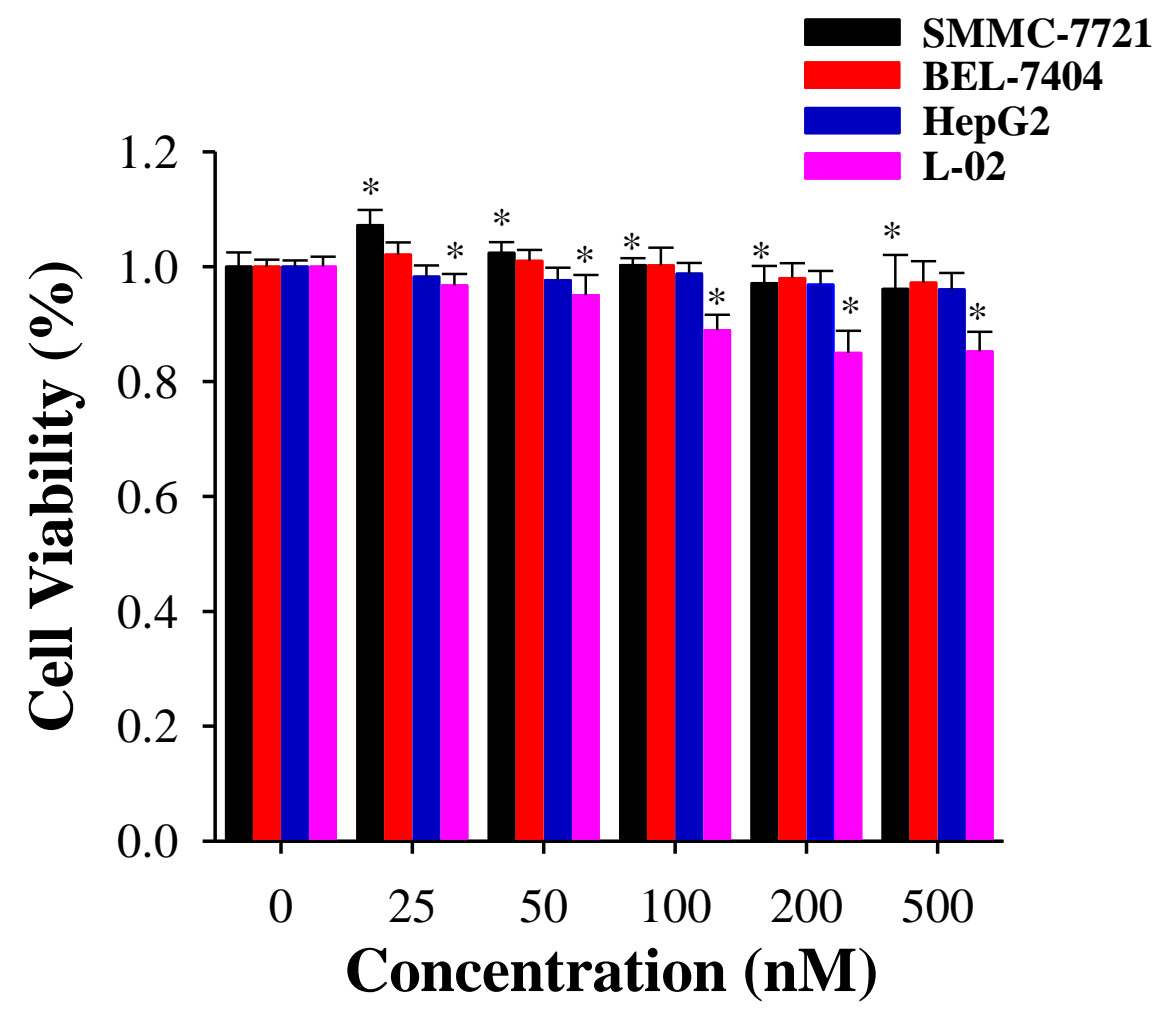

Figure S5. Viability assay for SMMC-7721, BEL-7404, HepG2 and L-02 cells treated with the $3 \mathrm{D}$ catcher probe of various concentrations $(0 \mathrm{nM}, 25 \mathrm{nM}, 50 \mathrm{nM}, 100 \mathrm{nM}$, $200 \mathrm{nM}, 500 \mathrm{nM})$ with incubation time $24 \mathrm{~h}$ at $37^{\circ} \mathrm{C} .\left(^{*}, P<0.05\right)$ 


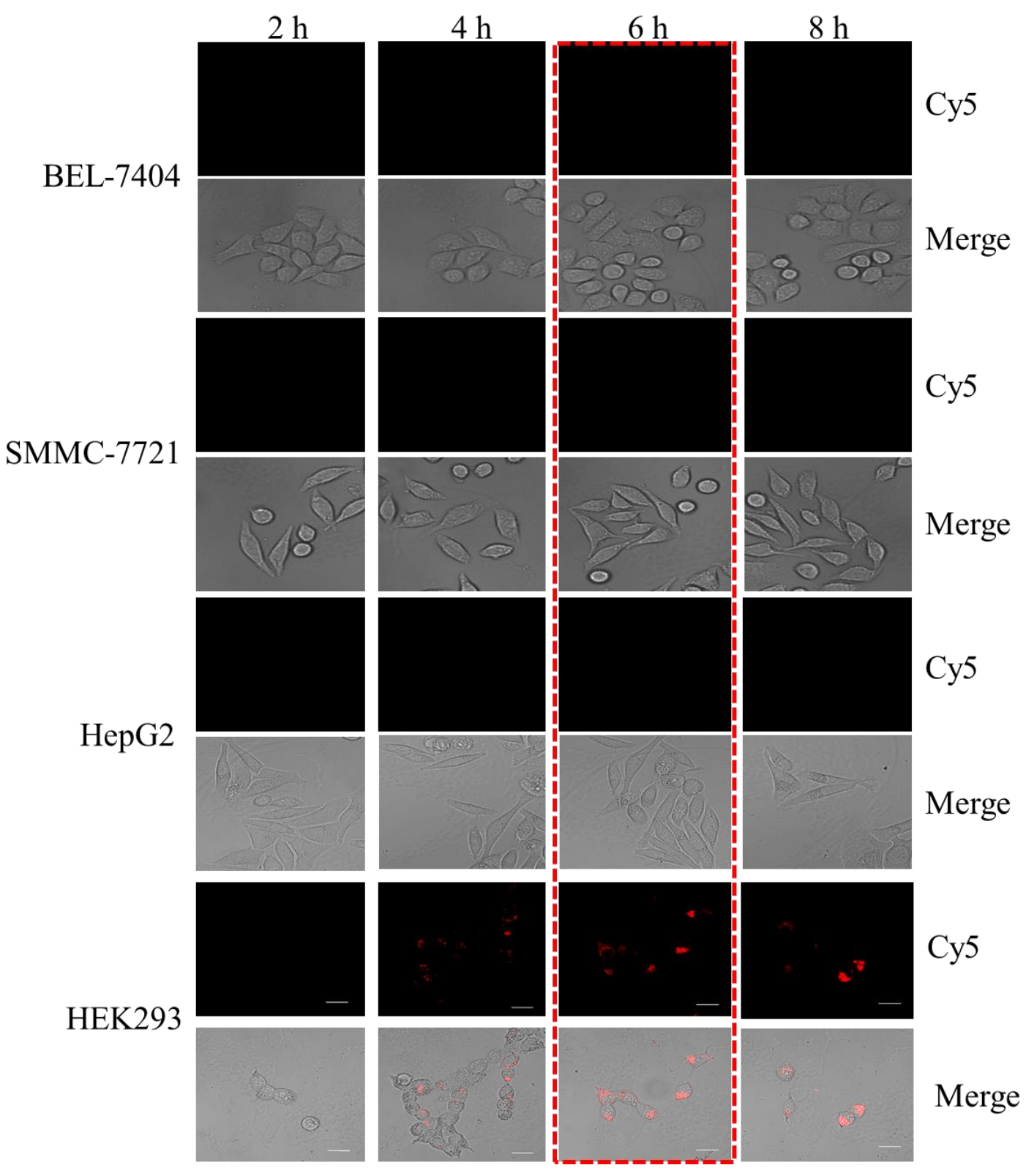

Figure S6. Confocal images of different cell types (HEK293, SMMC-7721, HepG2, BEL-7404) treated with $3 \mathrm{D}$ catcher $(50 \mathrm{nM})$ for $2,4,6$, and $8 \mathrm{~h}$ at $37^{\circ} \mathrm{C}$, respectively. The red fluorescence signals were recorded using Cy5 in the green channel from $655 \sim 780 \mathrm{~nm}$. Scale bar $=20 \mu \mathrm{m}$. 